\title{
Building Result-Based Accountability in an Organization
}

\author{
Abu-Hussain Jamal, Mohammad Essawi, Oleg Tilchin* \\ Al-Qasemi Academic College of Education, Baqa El-Gharbieh, Israel \\ Email: "otilchyn@yahoo.com
}

Received 10 May 2014; revised 12 June 2014; accepted 2 July 2014

Copyright (C) 2014 by authors and Scientific Research Publishing Inc.

This work is licensed under the Creative Commons Attribution International License (CC BY). http://creativecommons.org/licenses/by/4.0/

(c) (i) 0 pen Access

\section{Abstract}

The suggested approach can serve as a guide for building accountability, which promotes successful attainment of a desired organizational goal. The approach provides for the involvement of the largest possible number of employees working toward a result. First, a result structure for attaining an organizational goal is formed through the establishment of interrelation between corresponding tasks, determined by detailed elaboration of the sequential actions. Next, an accountability structure combining accountability levels, structural measures of accountability for the results of performing tasks, and skills relevant to each task is created. Third, the accountability structure and the reward conditions for acceptance of accountability for task performance are declared in an organization. Fourth, the tasks to be performed by employees are chosen, for which employees are to be held accountable as task performers or as task collaborators. The choice is realized by examination of the accountability structure and self-assessment of employee skills. Accountability acceptance is adjusted by the limitations. Fifth, the individual choices of employees are coordinated through discussion among employees with the participation of managers. As a result, heterogeneous collaborative teams to perform the tasks are formed through all levels organizational structure. Finally, building result-based accountability in an organization is completed through the flexible rewarding of employees for accountability acceptance. The reward is divided according to principle: The bigger the measure of accepted accountability is, the greater the reward the employees receive. The flexibility of reward correlates to adjustment of the size of the reward with regard to the roles of employees in task performance and the accountability measure of the employees.

\section{Keywords}

Building Accountability, Result Structure, Accountability Structure

\footnotetext{
${ }^{*}$ Corresponding author.
} 


\section{Introduction}

Accountability is one of the most fundamental, crucial, and dynamic factors affecting an organization's success [1]. According to Webster's Dictionary, accountability can be determined as an obligation or willingness to accept responsibility for actions. Accountability is "clear commitments that-in the eyes of others-have been kept” [2]. Accountability means that employees can rely on one another to keep performance commitments [3]. Accountability should serve as a constitutive element of a successful organization strategy [2].

An employee should take personal accountability for concrete results. It gives an employee a sense of purpose and leads to improved performance over improved processes [2] [4]. The result of performance is influenced by choices, behaviours, and actions of an employee. Result-based accountability produces measurable improvements of an organization's results through embedding outcome-based decision making into organizational processes [5]. Therefore, a purposeful collective impact on an organization's activity is achieved through resultbased accountability.

Suitable result-based accountability engenders better relationships among employees, and improves job satisfaction and performance [2]. It is caused by the willingness and desire of an employee to take accountability for the required result. Willingness is an intellectual component of accountability [6]. It characterizes the presence of the employee's skills for the job directed towards attaining the required result. Desire for taking accountability for the result is incited by the creation of a favourable organizational environment, and is consistent with his (her) desired outcomes [7].

The favourable organizational environment for acceptance of suitable accountability is created by leadership's cultivation of an accountability culture [3]. An accountability culture should inspire accountability through creating and sustaining purposefulness, fulfillment, and motivation of employees, and by building trust and collaboration among them [6] [8]. The accountability culture promotes greater performance, and higher quality is characterized by the presence of dynamic teams through all levels of an organizational structure, with trustworthy communications between employees and managers [8]. The team members hold themselves mutually accountable for outcomes [9]. The team accepts accountability for the results of its actions [10].

The embodiment of result-based accountability process intends first building accountability and then performance accountability [5]. Building accountability should take into consideration the mutually influential behaviors of employees that affect the accountability process. It requires, first of all, aligning employees with business strategy [11]. Performance accountability is continually asking "How I doing?" [2]. Attainment of the result is assessed by the use of performance measurements. Thereby, embodiment of result-based accountability provides for the success of an organization when "the right things are done, the right way, by the right staff, at the right time" [8].

The primary stage of the accountability process is building accountability. The goal of this paper is to develop an approach for building accountability in an organization.

\section{Literature Review}

Research of different aspects of an accountability process in an organization is examined.

Bergsteiner [11] created an accountability theory that describes and explains the accountability process and factors in influencing the process. On the basis of the theory, the author designed a Holistic Accountability Model that shapes mutual influence and behaviors of employees in the accountability process. He ascertained the relationship of accountability and organizational culture. The author proposed a graphic system for producing accountability relationships in an organization, and a decision tree model for building goal-oriented accountability.

Schlenker [1] suggested the triangle model describing three crucial aspects of personal responsibility. These aspects are prescription (what is supposed to be done), identity (the sense of self), and an event (that is relevant to the prescription). The author defines three factors that influence the reduction of responsibilities for events: lack of connection between the prescription and identity; lack of connection between identity and the characteristics of the event, and weakening of the connection between the prescription and the event.

Kraines [12] introduced fixed accountability and relative accountability. Fixed accountability includes obligations of the employee to produce results and to use resources and processes as determined. Employees must observe commitments to deliver a result and to work according to managerial guidelines. Relative accountability intends: attaining a better result than prescribed by the employee role; fitting for purpose; using resources effec- 
tively; providing teamwork.

Frink \& Kimosky [13] affirmed that since employees are interdependent at work, they must interact with each other according to their roles in organizational activity. Accountability of employees should depend on their roles. The authors offer using role theory as a framework for accountability. They stressed the need to coordinate employee actions in order to provide organizational effectiveness.

Samuel \& Chiche [7] determined accountability as action that's consistent with the desired outcomes of an employee. The authors have developed the personal accountability model, furthering realization of the intention to stay accountable. The model components are: recognize, own, forgive, self-examine, learn, and take action. Grimshaw \& Baron [14] suggested the three conditions of providing accountability. The conditions are to communicate distinct requirements for performance to employees; to create a structure of influence according to desired performance, and lead discussion among employees directed towards coordination their possibilities.

Connors \& Smith [15] considered creation of accountability culture in an organization as a result of consecutive realization of the steps conducive to employee accountability. These steps are: see it, own it, solve it, and do it. Essawi [16] created the structured dynamic value confrontation leadership model for organizational culture change. The model induces employees to take accountability for changing their cultural values according to the required organizational values.

Samuel [3] offered a systematic approach to increasing personal and organizational accountability. The approach includes the accountability culture elements and the leadership strategies directed towards development of the accountability culture. The elements are: clear intention determining the desired result and deadline its completion; interdependence of the employees to produce the desired result; effective execution is caused by coordination, timing, communication, and actions of the employees necessary to attain the desired result; liquidation of dysfunctional habits; responsive recovery; the measuring of results for providing good performance, and rewarding. The author's proposed leadership strategy involves creating a clear direction of organizational activity; building a plan that guarantees the result; communicating to influence; executing to realize high performance, and developing employees to be highly accountable performers.

Building accountability intends the need to form teams of employees. Team efficiency is characterized by a state of collaboration of team members. Evans (2008) claimed success of an organization requires embedding accountability into its strategy, determining clear expectations, and creating relationships among team members based on accountability. Owing to this, job satisfaction and organizational performance are improved.

Essawi \& Tilchin [17] presented an adaptive collaboration (AC) environment. The environment is formed by the integration of a dynamic team-based structure of an organization and a structure of the changes. Every change is conducted by performing suitable interrelated tasks. Adaptability of the collaborative environment is expressed by adjusting an organizational structure and a structure of tasks to dynamics of changes. The conditions of formation of teams and the order of allocation of members of teams for performance of tasks providing effective collaborative interactions are defined. The authors developed the DOCA model to shape the AC environment. The model realizes: determining an infrastructure of AC; organizing AC; creating AC groups; assigning collaborative group members to perform the tasks required to conduct adaptive changes.

Abu-Hussain, Essawi \& Tilchin [18] developed an approach to enhancing the Project-Based Collaborative Learning (PBCL) model through building result-based accountability. Enhancing the model is resulted in organizing PBCL as a two-phase process of building accountability for learning results, with specific formation of a knowledge heterogeneous collaborative group of students, and the design of a flexible assessment system. Student accountability for performance of determined project tasks is built through self-assessment of knowledge. A collaborative group is formed as a result of the coordination of personal accountabilities of students, taking into account teacher requirements providing students' shared accountability in performance of a group project.

The analysis of the above publications shows that the challenges associated with building result-based accountability in an organization remain. An organizational environment that induces the building accountability of employees for common performance goals has not been created. Fitting accountability of employees to organizational needs has not been provided. Measure accountability for tasks performance has not been dependent on order of delivering results. Self-managing teams of employees through all levels of organizational structure have not been formed. Sharing accountability for results gaining distinction of employee skills has not been specified. A balance between competition and collaboration among employees for receiving results of task performance has not been achieved. Rewarding employees has not been connected with the measure of their accountability for results. 


\section{Building Result-Based Accountability in an Organization}

The review of research allows for the conclusion that result-based accountability of employees can serve as a constructive mean for attaining a desired organizational goal. The proposed approach is aimed at building effective result-based accountability of employees by taking into consideration of employee skills and interdependence of task performance of which leads to the desired organizational goal.

The purpose of the approach is reached through: determination of the required organizational goal; forming the result structure leading to the goal; determination of levels and a structural measure of accountability for delivering the results; accepting accountability of employees for results through self-evaluation their willingness and desire to perform suitable tasks, and creating the organizational environment that fosters building effective result-based accountability.

The approach intends consistent performance of the determined steps.

\section{Step1: Forming a result structure for attaining a desired organizational goal}

At first, the actions for attaining a desired organizational goal (an organizational result) and their sequence are developed by managers. Each following action in the action sequence is more complicated. It allows introduction of complexity levels of the actions. A set of tasks realizing each action is formed. Consequently, an aggregate of task sets corresponds with the set of actions. The skills are required for a task performance. These are the task-relevant skills. Performance of the tasks corresponding with different actions requires different ordered skills. The result of performing each task is determined.

If the result of performance of task $z_{i}$ is needed for performance of task $z_{j}$, then task $z_{j}$ depends on task $z_{i}$. A task structure (a work breakdown structure) is built by taken into consideration of task dependencies. Thereby, the suitable interrelated tasks of the task structure correspond to each action. The task structure engenders the result structure since the results are received by performance of the tasks. Hence, the result structure is a set of the interrelated results leading to the desired organizational goal. The result of performance of the task which belongs to the last action from the action sequence and hasn't following tasks is a final result of the result structure.

\section{Example 1:}

The result structure leading to the desired organizational goal is represented by Figure 1.

The actions, the complexity levels of the actions, the interrelated tasks realizing the corresponding actions, and task-relevant skills for each task are signed by Figure 1. The task performance results are shown with arrows. The performance result of the task $\mathrm{z}_{8}$ is the final result of the result structure.

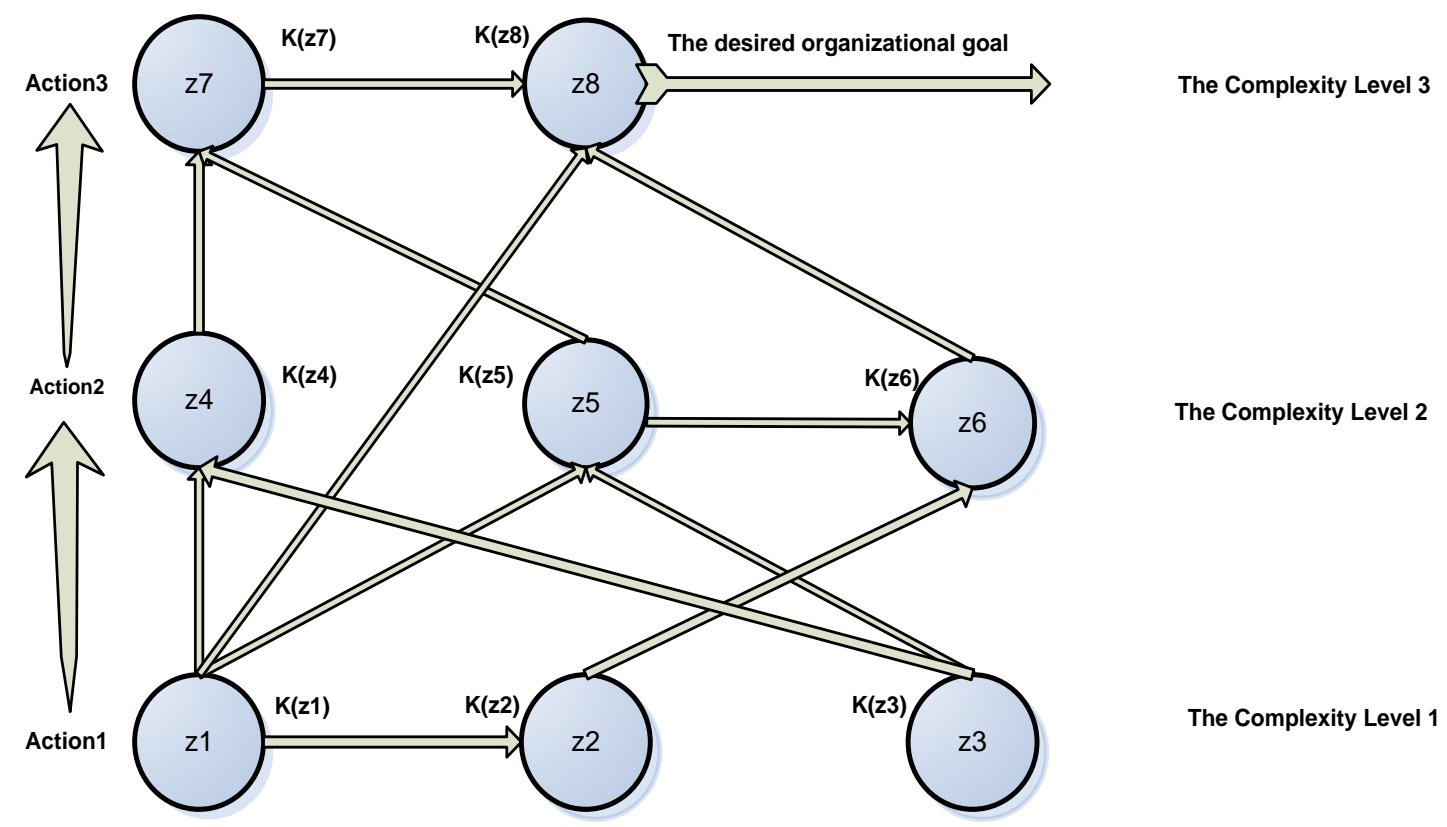

Figure 1. The result structure for attaining the desired organizational goal. 


\section{Step 2: Building an accountability structure}

A result structure determines the order of delivering the results of task performance. Furthermore, it is ordered according to the actions' performance complexity. Performance complexity from the task realizing the action corresponds with its performance complexity. Then, a level of accountability for results of performing the tasks can be defined as one corresponding with a level of action complexity.

A task, result performance of which is needed for performance of others tasks, can be named a providing task. A performance result of a providing task is required for performance of other tasks which can be named successsors of a task. Obviously, accountability for a result of performance of a providing task should depend on performance complexity of its successors, which cannot be performed without delivering this result. Consequently, structural measure of accountability for the result of task performance can be introduced. It equals the sum of the performance complexities of a task's successors. The accountability measure for the performance result of a task is determined as the sum of task performance complexity and structural measure of accountability for result of its performance. Hence, accountability measures for the task's performance determine accountability structure on the set of interrelated results.

\section{Example 2:}

Accountability measure for performance task $z_{1}$ equals 9 , since the task $z_{1}$ corresponds with the first accountability level, and structural measure of its accountability equals 8 . The task $\mathrm{z}_{1}$ has four successors: the tasks $\mathrm{z}_{2}$, $\mathrm{z}_{4}, \mathrm{z}_{5}$, and $\mathrm{z}_{8}$. Complexity of the task $\mathrm{z}_{2}$ is one (the task corresponds with the first accountability level). Complexity of each from the tasks $z_{4}$ and $z_{5}$ is two (the tasks correspond with the second accountability level). Complexity of the task $z_{8}$ is three (the task corresponds with the third accountability level). Accountability measure for performing the task $\mathrm{z}_{7}$ equals 6 , since the task $\mathrm{z}_{7}$ corresponds with the third accountability level and has one task successor of the same accountability level. The accountability structure is presented by Figure 2.

The calculated measures of accountability for results are marked near the tasks.

\section{Step 3: Declaration of the accountability structure and the reward conditions}

The accountability structure is manifested in the organization for employees' awareness. The employees are informed about the need to evaluate their possibility to participate in performance of the tasks.

Aspiration to induce the employees toward productive activity conducive to the desired organizational goal causes a declaration of the reward conditions. Prior familiarity of employees with the reward conditions is provided. The conditions foresee rewarding all employees, expressing intention to participate in the further performance of the tasks. The reward conditions take into account the possible roles of an employee in performance of the tasks. The roles are task performer, and task collaborator.

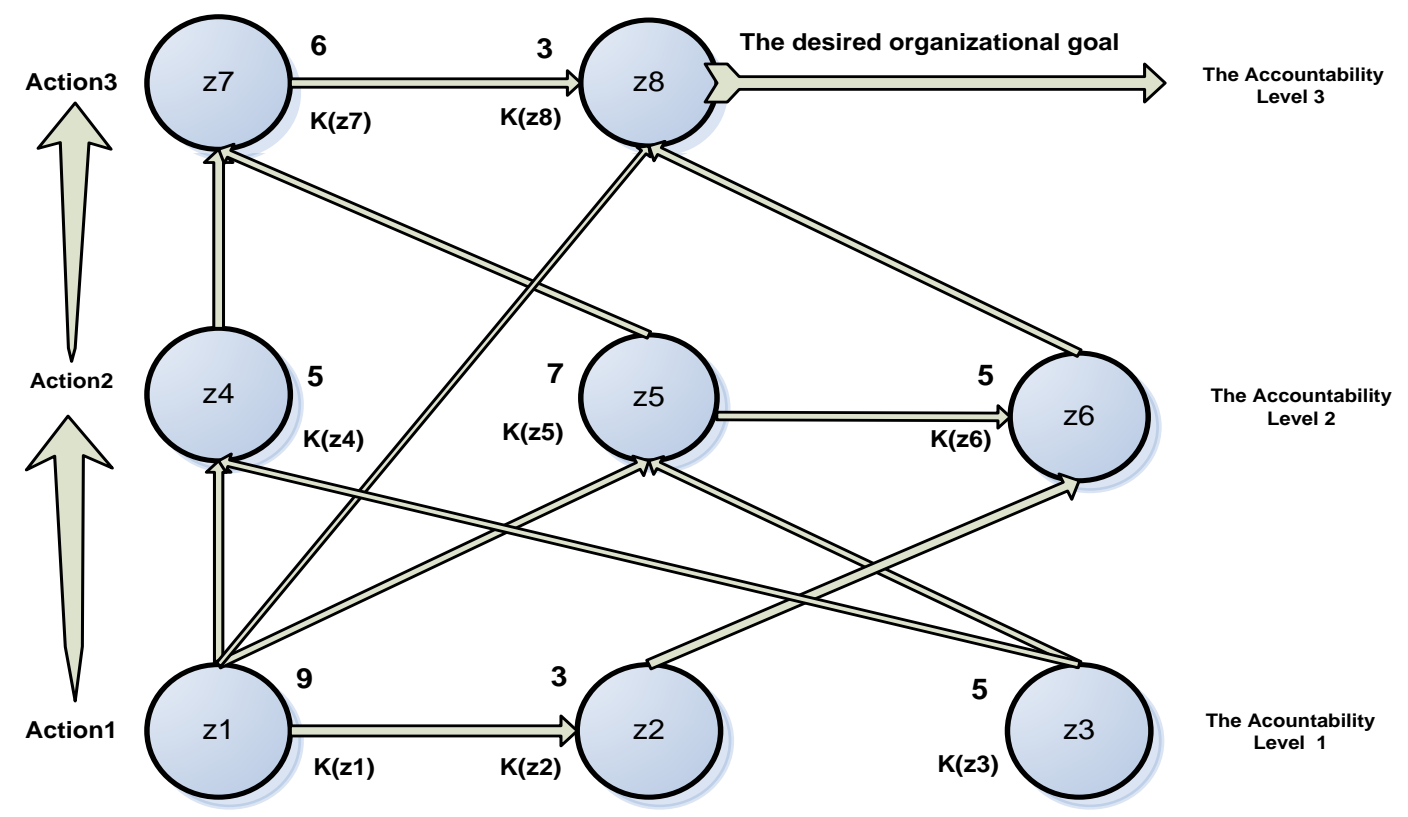

Figure 2. The accountability structure. 


\section{Step 4: Self-assessment of employees}

An employee examines the accountability structure and realizes self-assessment of his (her) willingness to perform the tasks. During self-assessment an employee compares his (her) skills with a task-relevant skills. As a result of self-assessment, an employee expresses a sound desire to be accountable for task performance, or to share accountability for task performance through collaboration with other employees. The willingness to be accountable for task performance supposes declaration (substantiation) by an employee of availability of the most skills needed for successful performance of the chosen task. The willingness of an employee to collaborate in task performance is the result of a small quantity of available skills corresponding to task-relevant skills.

The aspiration to provide qualitative and timely performance of the tasks requires from the managers an introduction of the limitations. An employee should follow the limitations during the self-assessment process. The limitations are the quantity of the tasks for the performance of which an employee may be accountable, and the quantity of the tasks in performance of which an employee may collaborate. An employee should choose the related tasks for taking accountability or in sharing accountability for performance.

A table containing results of self-assessment of employees' willingness to perform the tasks can be created. The rows of the table correspond to staff and divisions of organizational structure to which they belong. The columns of the table correspond to the tasks that should be performed. Task-relevant skills (K), accountability measure for results of a task performance, and successors of a task are presented in a heading of a column. Intersection of a row and a column contains the role is chosen by an employee owing to self-assessment. The roles are task performer $(\mathrm{P})$, and task collaborator $(\mathrm{C})$.

\section{Example 3:}

The measures of accountability for the results of performing the tasks and the successors of the tasks are caused by the accountability structure (Figure 2). During the self assessment process the employees should follow the limitations: quantity of the tasks for performance of which an employee may be accountable isn't more then two; quantity of the tasks, in performance of which an employee may collaborate isn't more than three. The outcomes of employees' self-assessments are presented by Table 1 . Thus, the employee $\mathrm{e}_{1}$ is willing to take accountability for the results of performing the task $\mathrm{z}_{1}$, and to share accountability through collaboration of performance of the tasks $\mathrm{z}_{2}, \mathrm{z}_{4}$, and $\mathrm{z}_{5}$.

Self-assessments of employees are directed towards attaining a balance between competition and collaboration among employees. If an employee doesn't have sufficient skills for task performance, he (she) can't compete with peers, but can make a decision to collaborate.

If an employee has needed skills for task performance, he (she) competes with his (her) peers for performance of this task. Yet, he (she) can also collaborate with other employees for performance of other tasks. Furthermore, through self-assessment, the employees choose their roles in performance of the tasks.

\section{Step 5: Coordination of self-assessment outcomes}

Building accountability for results intends the observance of aforementioned limitations and satisfying the specific requirements in forming a heterogeneous collaborative group for task performance. One collaborative group should be formed for task performance. Heterogeneity of a collaborative group is caused by difference of the employees' skills.

The requirements are:

Table 1. Self-assessments of the employees.

\begin{tabular}{cccccccccc}
\hline & \multirow{2}{*}{$\begin{array}{c}\text { The Divisions } \\
\text { The Employees }\end{array}$} & $\begin{array}{c}\mathrm{z}_{1} \mathrm{~K}_{1} \\
\text { of Organizational }\end{array}$ & $\mathrm{z}_{2} \mathrm{~K}_{2}$ & $\mathrm{z}_{3} \mathrm{~K}_{3}$ & $\mathrm{z}_{4} \mathrm{~K}_{4}$ & $\mathrm{z}_{5} \mathrm{~K}_{5}$ & $\mathrm{z}_{6} \mathrm{~K}_{6}$ & $\mathrm{z}_{7} \mathrm{~K}_{7}$ & $\mathrm{z}_{8} \mathrm{~K}_{8}$ \\
\hline & Structure & 9 & 3 & 5 & 5 & 7 & 5 & 6 & 3 \\
\hline $\mathrm{e}_{1}$ & $\mathrm{~d}_{1}$ & $\mathrm{P}$ & $\mathrm{C}$ & & $\mathrm{C}$ & $\mathrm{C}$ & & & \\
$\mathrm{z}_{2} \mathrm{z}_{5} \mathrm{z}_{8} \mathrm{z}_{4}$ & $\mathrm{z}_{6}$ & $\mathrm{z}_{4} \mathrm{Z}_{5}$ & $\mathrm{z}_{7}$ & $\mathrm{z}_{6} \mathrm{Z}_{7}$ & $\mathrm{z}_{8}$ & $\mathrm{z}_{8}$ & - \\
$\mathrm{e}_{3}$ & $\mathrm{~d}_{2}$ & $\mathrm{C}$ & $\mathrm{C}$ & & $\mathrm{P}$ & $\mathrm{C}$ & & $\mathrm{P}$ & \\
$\mathrm{e}_{4}$ & $\mathrm{~d}_{3}$ & $\mathrm{C}$ & $\mathrm{P}$ & & & & $\mathrm{P}$ & & $\mathrm{C}$ \\
$\mathrm{e}_{5}$ & $\mathrm{~d}_{4}$ & & & $\mathrm{P}$ & $\mathrm{C}$ & & & $\mathrm{C}$ & \\
$\mathrm{d}$ & $\mathrm{d}_{4}$ & $\mathrm{P}$ & & & & $\mathrm{P}$ & $\mathrm{C}$ & $\mathrm{C}$ & \\
\hline
\end{tabular}


- One member of a collaborative group should be accountable personally for the result of task performance

- The collaborative group members should have necessary skills for a task performance

- Mutual addition of skills of the collaborative group members should be provided

- Maximal allowed quantity of employees in a collaborative group should be fixed

The table containing the self-assessment outcomes of the employees is manifested in an organization. The objective of manifestation is to foster coordination of the self-assessment outcomes to complete the building of accountability of employees for the results of the tasks performed. Coordination is realized through discussion of employees with participation of managers. During discussion, every employee compares tasks for which he would be accountable with those tasks chosen by peers and the performance for which they would be accountable. The aim of comparison is to coordinate taking personal accountability and sharing accountability for performance of the tasks.

The tasks selected earlier by an employee can be replaced by other tasks due to coordination with peers. If some employees want to be accountable for the result of performance of the same task, despite coordination of their outcomes, then the managers determine the employee who should be accountable for the result. All tasks should be performed. Hence, if there is not an employee who shows willingness to accept accountability for the result of a task performance, the managers delegate accountability to the most suitable employee. Hence, forming heterogeneous collaborative groups leading to the building accountability of employees for results of the tasks performance through compared analysis of employees' choices is realized. The collaborative group members share accountability for the result with the task performer.

\section{Example 4:}

Analysis of the self-assessment outcomes of employees (Table 1) allows for the conclusion that requirements for forming collaborative groups for task performance are not observed. The employee $e_{1}$ and the employee $e_{5}$ are competing for taking of accountability for the result of task $z_{1}$ performance. One employee $e_{3}$, chose a role of the task $\mathrm{z}_{8}$ collaborator; another employee $\mathrm{e}_{4}$, has the role of the task $\mathrm{z}_{3}$ performer.

Coordination of self-assessment outcomes between the employee $e_{1}$ and the employee $e_{5}$ through discussion with the participation of managers is realized. Therefore, the employee $e_{5}$ accepted accountability for result of the task $z_{8}$ instead of the task $z_{1}$. A comparison of skills of employee $e_{4}$ with task $z_{8}$ relevant skills allowed this employee to take the role of collaborator in the performance of task $z_{8}$. There is also possibility for employee $e_{5}$ to take the role of collaborator in performance of task $z_{3}$. The changes are presented by Table 2 that reflects the outcomes of building accountability of employees following the results of task performance. The outcomes satisfy the fixed limitations and the requirements, and represent built accountability of the employees for the results of performance of the tasks.

Step 6: Flexible rewarding of employees for accountability acceptance

Established rewarding of employees for acceptance of accountability is divided according to the measure of their accountability taken for the corresponding result: the bigger measure of accountability for the result, the greater reward the employees receive.

Example 5:

The established size of employees' rewards equals $\$ 1290$. There are eight tasks $z_{1}, z_{2}, \ldots, z_{8}$. Accountability measures for performance of the tasks are $9,3,5,5,7,5,6,3$, accordingly (Table 2). Then, distribution of re-

Table 2. Building accountability outcomes.

\begin{tabular}{|c|c|c|c|c|c|c|c|c|c|}
\hline \multirow{3}{*}{ Employees } & \multirow{3}{*}{$\begin{array}{c}\text { The Divisions } \\
\text { of Organizational } \\
\text { Structure }\end{array}$} & $\mathrm{z}_{1} \mathrm{~K}_{1}$ & $\mathrm{z}_{2} \mathrm{~K}_{2}$ & $\mathrm{z}_{3} \mathrm{~K}_{3}$ & $\mathrm{z}_{4} \mathrm{~K}_{4}$ & $\mathrm{z}_{5} \mathrm{~K}_{5}$ & $\mathrm{z}_{6} \mathrm{~K}_{6}$ & $\mathrm{z}_{7} \mathrm{~K}_{7}$ & $\mathrm{z}_{8} \mathrm{~K}_{8}$ \\
\hline & & 9 & 3 & 5 & 5 & 7 & 5 & 6 & 3 \\
\hline & & $\mathrm{z}_{2} \mathrm{Z}_{4} \mathrm{Z}_{5} \mathrm{Z}_{8}$ & $\mathrm{z}_{6}$ & $\mathrm{z}_{4} \mathrm{Z}_{5}$ & $\mathrm{Z}_{7}$ & $\mathrm{z}_{6} \mathrm{z}_{7}$ & $\mathrm{z}_{8}$ & $\mathrm{z}_{8}$ & - \\
\hline $\mathrm{e}_{1}$ & $\mathrm{~d}_{1}$ & $\mathrm{P}$ & C & & C & C & & & \\
\hline $\mathrm{e}_{2}$ & $\mathrm{~d}_{2}$ & C & C & & $\mathrm{P}$ & C & & $\mathrm{P}$ & \\
\hline $\mathrm{e}_{3}$ & $\mathrm{~d}_{3}$ & $\mathrm{C}$ & $\mathrm{P}$ & & & & $\mathrm{P}$ & & $\mathrm{C}$ \\
\hline $\mathrm{e}_{4}$ & $\mathrm{~d}_{4}$ & & & $\mathrm{P}$ & C & & & C & $\mathrm{C}$ \\
\hline $\mathrm{e}_{5}$ & $\mathrm{~d}_{4}$ & & & C & & $\mathrm{P}$ & $\mathrm{C}$ & $\mathrm{C}$ & $\mathrm{P}$ \\
\hline
\end{tabular}


ward among the employees for accountability acceptance for performance of the tasks results in $\$ 270, \$ 90, \$ 150$, \$150, \$210, \$150, \$180, \$90, accordingly.

The predetermined size of reward for accountability acceptance for performance of the tasks is divided between employees on the basis of their roles in task performance, e.g. task performer receives $80 \%$ and task collaborators receive $20 \%$ of the reward. The task collaborators reward is divided among them in equal parts.

\section{Example 6:}

The employees' reward for accountability acceptance for performance of task $z_{1}$ equals $\$ 270$ (Example 5). According to Table 2, the employee $\mathrm{e}_{1}$ is the performer of this task, and the employee $\mathrm{e}_{2}$ and the employee $\mathrm{e}_{3}$ are the task $\mathrm{z}_{1}$ collaborators. Then, rewarding the task performer equals $\$ 216$, and rewarding the task collaborators equals $\$ 54$.

An integral personal award for an employee is calculated by the summation of his awards as a performer and collaborator in corresponding tasks.

Flexibility of rewarding is attained owing to adjustment of the size of reward with regard to the measure of an employees' accountability for results (an employee which accepts greater accountability receives a greater reward) and the roles of employees in task performance.

\section{Conclusions}

The suggested approach allow building result-based accountability in an organization through forming an accountability structure, self-assessment of an employee's willingness to take accountability for delivering the results, coordination of self-assessment outcomes, and inducing desire of an employee for taking accountability.

An accountability structure is determined by the accountability measure for the result of task performance. The accountability measure is calculated as the sum of task performance complexity and the structural measure of accountability for the result of its performance. Task performance complexity equals performance complexity of a corresponding action. Structural measure of accountability of task performance is based on a structure of delivering results conducive to a desired goal. It equals the sum of performance complexities of task successors. Owing to that, dependence of measure accountability for task performance upon order of delivering results has been provided.

Self-assessment of an employee's willingness to take accountability for delivering results is aimed at choice of tasks by the employee, and the roles in their performance through comparison of personal skills with task-relevant skills. The aspiration to encourage individual choice (promoting qualitative and timely performance of the tasks) creates the introduction of the limitations that adjust self-assessment process.

Coordination of self-assessment outcomes of employees through discussion with the participation of managers completes the building accountability for results. It is adjusted by the specific requirements of forming a team, gaining distinction for employee skills for task performance. The coordination allows formation of selfmanaging teams of employees through all levels of organizational structure and provides sharing accountability for results. Due to this coordination, a balance between competition and collaboration among employees for results of tasks performance is achieved.

Desire of an employee to take accountability is induced by prior declaration of the reward conditions and the use of flexible rewards. Flexibility of rewarding is attained owing to adjustment of the size of the reward with regard to the measure of an employees' accountability for results (an employee which takes greater accountability receives a greater reward), and the roles of employees in task performance.

\section{References}

[1] Schlenker, B.R. (1997) Personal Responsibility. Applications of the Triangle Model. In: Cummings, L. and Staw, B., Eds., Research in Organizational Behavior, JAI Press, Greenwich, 241-301.

[2] Evans, H.J. (2008) Winning with Accountability: The Secret Language of High-Performing Organizations. Corner Stone Leadership Institute, Dallas.

[3] Samuel, M. (2001) The Accountability Revolution: Achieve Breakthrough Results in Half the Time. 2nd Edition, Facts on Demand Press, Tempe.

[4] Samuel, M. (2012) Making Yourself Indispensable: The Power of Personal Accountability. Portfolio Hardcover, New York.

[5] Mark, F. (2009) Trying Hard Is Not Good Enough. Book Surge Publishing, Victoria. 
[6] Bustin, G. (2014) Accountability: The Key to Driving a High-Performance Culture. McGraw-Hill, New York.

[7] Mark, S. and Sophie, C. (2004) The Power of Personal Accountability: Achieve What Matters to You. Xephor Press, New York.

[8] Worral, D. (2013) Accountability Leadership: How Great Leaders Build a High Performance Culture of Accountability and Responsibility. Worrall \& Associates, Carlton.

[9] Katzenbach, J.R. and Smith, D.K. (2006) The Wisdom of Teams: Creating the High-Performance Organization (Collins Business Essentials). Harper Business, New York.

[10] Browning, H. (2012) Accountability: Taking Ownership of Your Responsibility. Pfeiffer, Greensboro.

[11] Bergsteiner, H. (2012) Accountability Theory Meets Accountability Practice. Emerald Group Publishing Limited, Bingley.

[12] Kraines, G. (2011) Accountability Leadership: How to Strengthen Productivity through Sound Managerial Leadership. The Career Press Inc., New York.

[13] Frink, D.D. and Kimoski, R.J. (2004) Advancing Accountability Theory and Practice. Introduction to the Human Resource Management Review Special Edition. Human Resource Management Review, 14, 1-17. http://dx.doi.org/10.1016/j.hrmr.2004.02.001

[14] Grimshaw, J. and Baron, G. (2010) Leadership without Excuses: How to Create Accountability and High Performance (Instead of Just Talking about It). McGraw-Hill, New York.

[15] Connors, R. and Smith, T. (2011) Change the Culture Change the Game. Penguin Group Inc., New York.

[16] Essawi, M. (2012) The Value Confrontation Leadership Model. International Leadership Journal, 4, 72-82. http://www.tesc.edu/documents/ILJ_Summer_2012.pdf

[17] Essawi, M. and Tilchin, O. (2012) Adaptive Collaboration Model for Organizational Change. American Journal of Industrial and Business Management, 2, 145-152. http://dx.doi.org/10.4236/ajibm.2012.24019

[18] Abu-Hussain, J., Essawi, M. and Tilchin, O. (2014) Accountability for Project-Based Collaborative Learning. International Journal of Higher Education, 3, 127-135. http://dx.doi.org/10.5430/ijhe.v3n1p127 
Scientific Research Publishing (SCIRP) is one of the largest Open Access journal publishers. It is currently publishing more than 200 open access, online, peer-reviewed journals covering a wide range of academic disciplines. SCIRP serves the worldwide academic communities and contributes to the progress and application of science with its publication.

Other selected journals from SCIRP are listed as below. Submit your manuscript to us via either submit@scirp.org or Online Submission Portal.
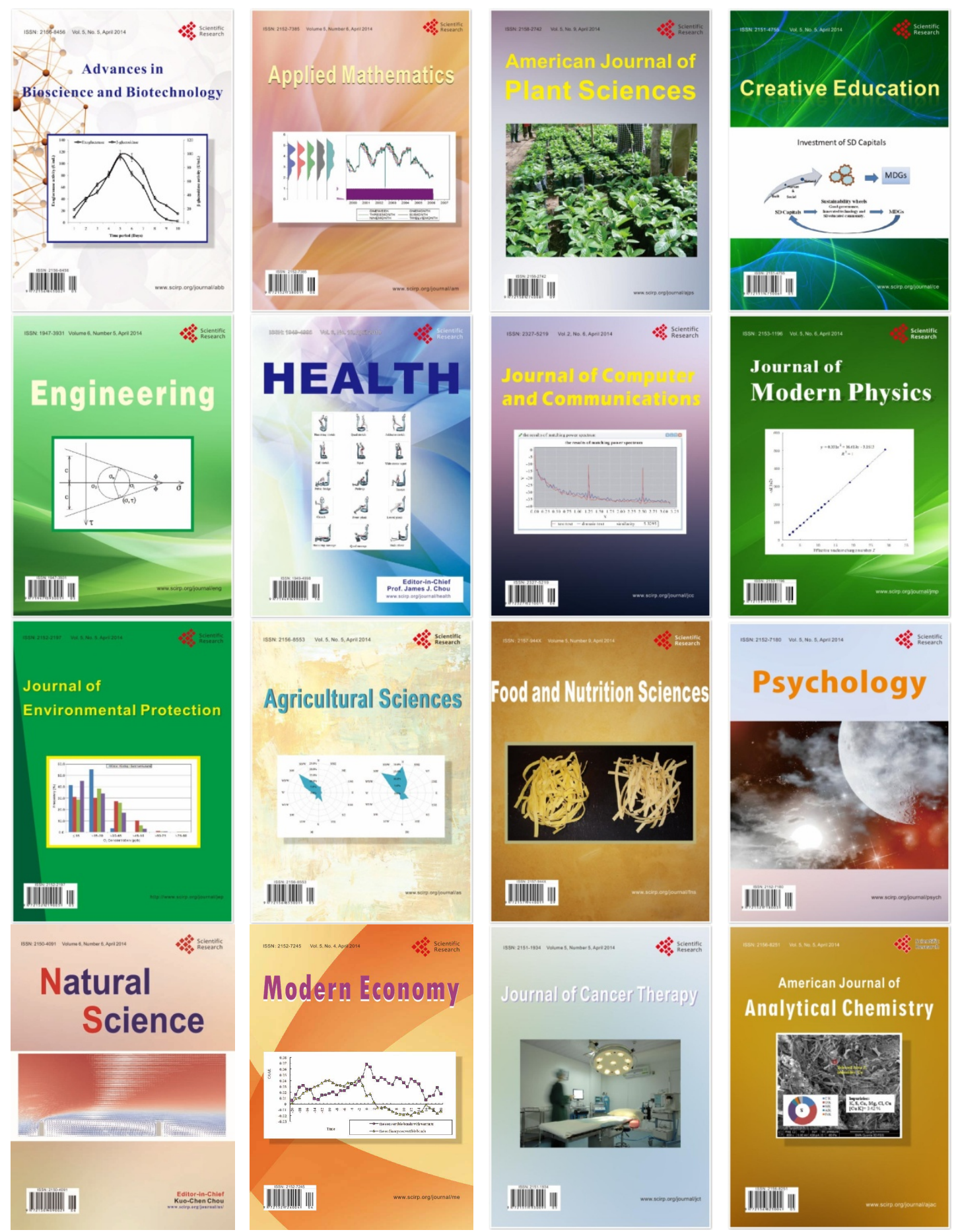